\title{
A fluorose dentária no Brasil: uma revisão crítica
}

\author{
Dental fluorosis in Brazil: \\ a critical review
}

Maria Cristina Teixeira Cangussu 1

Paulo Capel Narvai 2

Roberto Castellanos Fernandez 2

Valquíria Djehizian ${ }^{2}$

\footnotetext{
1 Departamento de Odontologia Social, Faculdade de Odontologia, Universidade Federal da Bahia. Rua Araújo Pinho 62, Salvador, $B A$

41110-150, Brasil. macristina@svn.com.br

2 Faculdade de Saúde Pública, Universidade de São Paulo.

Av. Dr. Arnaldo 715, São Paulo, SP 01246-904, Brasil. pcnarvai@usp.br djehizian@aol.com
}

Abstract This paper discusses dental fluorosis as a relevant public health problem, using a review of epidemiological studies published in the last 10 years on the disease's prevalence, severity, and risk factors. The results suggest that there are already more cases than expected, although few studies refer to major severity. Thus, measures are needed for the prevention and surveillance of dental fluorosis.

Key words Dental Fluorosis; Oral Health; Risk Factors

Resumo Discute-se neste trabalho a pertinência de se considerar a fluorose dentária como um problema de saúde pública no Brasil, utilizando como método a revisão dos estudos epidemiológicos publicados no país sobre prevalência e severidade da doença e dos seus possíveis fatores de risco. Os resultados sugerem que já são encontradas freqüências mais altas que as esperadas, embora com poucos casos de maior severidade. São necessárias, então, medidas de vigilância à saúde bucal.

Palavras-chave Fluorose Dentária; Saúde Bucal; Fatores de Risco 


\section{Introdução}

Na Odontologia, após a década de 80, pôde-se observar inúmeras mudanças nos padrões de morbidade das doenças bucais, decorrente de avanços nos processos de diagnóstico, mudanças nos hábitos sociais e de saúde, desenvolvimento industrial, cultural e difusão de técnicas preventivas, entre elas o uso maciço dos fluoretos (Mandel, 1992). O uso do flúor tem promovido melhorias significativas na saúde bucal e na qualidade de vida das populações, através da redução dos índices de cárie dental (Burt, 1995). Entretanto, inúmeros estudos têm sido divulgados identificando o primeiro sinal clínico do efeito tóxico dessa substância - a fluorose dentária (Brothwell \& Limeback, 1999; Burt, 1995; Fejerskov,1994).

Coloca-se então, a necessidade de avaliar criticamente os dados epidemiológicos existentes sobre a fluorose dentária, na perspectiva da mesma se constituir ou não num problema relevante em saúde pública. Além disso, se discutirá a efetividade e a segurança do uso do flúor em suas diversas apresentações, propondo ações de vigilância à saúde, de modo a garantir o seu máximo benefício sem efeitos colaterais indesejáveis.

\section{Fluorose dentária - aspectos clínicos e epidemiológicos}

A fluorose dentária origina-se da exposição do germe dentário, durante o seu processo de formação, a altas concentrações do íon flúor. Como conseqüência, tem-se defeitos de mineralização do esmalte, com severidade diretamente associada à quantidade ingerida. Geralmente, o aspecto clínico é de manchas opacas no esmalte, em dentes homólogos, até regiões amareladas ou castanhas em casos de alterações mais graves (DenBesten, 1999; Fejerskov, 1994).

Além da dosagem de flúor, outros fatores interferem na severidade da doença: baixo peso corporal, taxa de crescimento esquelético e períodos de remodelamento ósseo constituemse fases de maior absorção do flúor; estado nutricional, altitude e alterações da atividade renal e da homeostase do cálcio também são fatores relevantes (DenBesten, 1999). Nesse sentido, a doença é mais freqüente em dentes de mineralização tardia (dentição permanente) em crianças de baixo peso ou precário estado nutricional ou insuficiência renal crônica, sendo as faixas etárias da primeira e segunda infância consideradas as de maior risco à ingestão do flúor sistêmico e, conseqüentemente, seus efeitos maléficos (Fejerskov, 1994).
Os estudos epidemiológicos desenvolvidos no mundo na década de 90 (Tabela 1) descrevem diferenças na prevalência da fluorose, que variam desde a quase ausência da doença nas populações $-2,2 \%$, até proporções maiores que 90\% (Akpata et al., 1997; Downer, 1994). Em geral, as altas prevalências estão presentes onde existem fontes naturais com alto teor de flúor ou ingestão de múltiplas fontes desse íon e, historicamente, tem apresentado a fluorose de forma endêmica em todas as faixas etárias como a China (Burt, 1995), localidades na África (El Nadef \& Honkala, 1998; Kloss \& Haimanot, 1999; N’ang'a \& Valderhaug, 1993), Arábia Saudita (Akpata et al., 1997), Cingapura (Lo \& Bagramian, 1996), Estado Unidos, Canadá, Brasil e Colômbia (Azcurra et al., 1995; Clarck et al., 1994; Cortes et al., 1996; Selwitz et al., 1995; Skotowski et al., 1996).

A doença tem apresentado prevalências e severidade maiores em idades mais jovens em estudos num mesmo local, o que tem alertado a comunidade científica para a necessidade de um acompanhamento contínuo e efetivo, para a detecção de uma possível tendência de aumento secular da fluorose dental (Heintze et al.,1998; Levy et al., 1995). Entretanto, ainda existe discordância entre os achados: Selwitz et al. (1995) descrevem que na Suíça não foi encontrado nenhum indício de aumento na prevalência ou severidade da fluorose dentária, enquanto Lewis \& Banting (1994) identificam nos Estados Unidos um claro aumento de cerca de $33 \%$ da prevalência da doença em regiões com água fluoretada e de $9 \%$ sem água fluoretada acompanhadas de incremento na severidade.

No Brasil, não foram encontrados na literatura, estudos longitudinais que descrevem mudanças no perfil epidemiológico da fluorose dentária. Nos trabalhos publicados sobre a doença, têm-se prevalências entre zero (Campos et al., 1998) até 97,6\% (Capella et al., 1989), com grande variabilidade de acordo com as regiões (Tabela 2). Observa-se, entretanto, que mesmo com altas prevalências, a proporção de indivíduos que apresentam as formas moderada e severa ainda é pequena, só aumentando significativamente nos locais onde a fluorose é endêmica e deve-se à alta concentração do fluoreto nas fontes naturais de água.

A diversidade dos índices utilizados para a mensuração da doença, dificulta a comparabilidade dos estudos. Dentre eles têm-se o índice TF - Thylstrup e Fejerskov - (Fejerskov et al., 1994), que classifica a fluorose dentária em nove graus de severidade, e se propõe a precisar diferentes categorias de comprometimento do esmalte dentário nas formas mais graves, utili- 
Prevalência e severidade da fluorose dentária no mundo* em estudos publicados na década de 90.

\begin{tabular}{llcrr}
\hline Autor, ano de publicação & Local & Índice utilizado & Prevalência (\%) & Moderado-severo (\%) \\
\hline Azcurra et al. (1995) & Argentina & Dean & $52,0-78,0$ & 25,0 \\
Kloos et al. (1985) & Etiópia & Dean & $32,0-83,2$ & 48,2 \\
Lewis \& Banting (1994) & Estados Unidos & Dean & $12,2-51,2$ & $0,0-2,4$ \\
Clarck \& Berkowitz (1997) & Estados Unidos & TF & 46,0 & 3,0 \\
Skotowiski et al. (1996) & Estados Unidos & TSIF & 65,6 & 1,9 \\
Nowjack-Raymer (1995) & Estados Unidos & Dean & $6,9-9,0$ & $0,0-3,5$ \\
Clarck et al. (1994) & Canadá & TSIF & 60,0 & 0,1 \\
Marthaler (1996) & Suíça & TF & 38,0 & 0,0 \\
Downer (1994) & Inglaterra & Dean & $2,2-13,3$ & 0,0 \\
El Nadef et al. (1998) & Nigéria & Dean & $43,0-58,0$ & $2,0-3,0$ \\
Wang (1997) & Noruega & TF & $8,0-48,0$ & 0,0 \\
N'glang'a \& Valderhaug (1993) & Quênia & TF & $18,0-76,0$ & 1,5 \\
Lo \& Bragamian (1996) & Cingapura & Dean & $79,0-84,6$ & $29,8-37,9$ \\
Akpata et al. (1997) & Arábia Saudita & TF & $75,0-92,0$ & 44,7 \\
Kumar \& Swango (1999) & Estados Unidos & Dean & $7,9-14,4$ & $0,3-1,7$ \\
Selwitz et al. (1995) & Estados Unidos & TSIF & $15,3-66,7$ & $0,1-3,1$ \\
Jackson et al. (1995) & Estados Unidos & TSIF & $18,2-92,1$ & 25,1 \\
\hline
\end{tabular}

* todos os estudos utilizam a população de adolescentes ou escolares na coleta dos dados (6-17 anos de idade).

zando profilaxia prévia e secagem durante o exame clínico. Costuma ser mais indicado para populações com altas exposições a fluoretos ou alta prevalência da doença (Fejerskov, 1994; Gonini, 1999).

O índice de Dean é baseado em variações no aspecto estético do esmalte, desde normal/ questionável até a forma grave, abrangendo seis categorias. É um índice bastante utilizado, embora seja incapaz de descrever com clareza gradações importantes das formas mais severas da doença, já que estas estão agrupadas em uma única categoria (Fejerskov, 1994; Gonini, 1999). Já o índice TSIF, proposto por Horowitz em 1987, utiliza a superfície dental (oclusal, vestibular e lingual) como unidade de análise, classificando-as em 8 categorias, sendo do 1 a 3 variações nos graus de opacidade, enquanto de 4 a 8 graus de manchamento ou cavitação da estrutura (Gonini, 1999).

Apesar das diferenças entre os estudos, é de fundamental importância que os mesmos descrevam os casos de moderados a severos da doença. Estes são os considerados de importância para a saúde pública, pois provocam alterações estéticas e funcionais significativas, e percebidas como de relevância social para a comunidade (Clarck et al., 1994; Paiva et al., 1993).

$\mathrm{Na}$ Odontologia, utiliza-se como critérios de seleção dos problemas a magnitude, severidade e vulnerabilidade do dano à saúde, im- pacto potencial das medidas de controle, a transcendência social do problema e o grau de interferência na qualidade de vida dos indivíduos (Pinto, 1994). O ser humano, nessa perspectiva, passa a ser interpretado não só pelo seu aspecto físico, mas também mental, psicológico e estético.

A fluorose dental torna-se então um problema relevante em saúde pública, porque em suas formas moderada ou severa, provoca alterações funcionais e estéticas que interferem na formação da personalidade, na inserção no mercado de trabalho, exige tratamento odontológico de alta complexidade em casos mais graves, tem etiologia conhecida e é factível de ser prevenida.

Apesar de serem encontradas prevalências variáveis da fluorose dental em alguns municípios brasileiros, é sugestivo que exista a possibilidade de um maior risco do acometimento da população pela difusão maciça do uso dos fluoretos na atualidade (Clarck et al., 1994), especialmente os dentifrícios fluoretados, que tiveram o seu uso regulamentado e difundido no país somente na década de 90 (Villena et al., 1996). Assim, a fluorose dental se constitui numa doença importante para a adoção de ações de vigilância à saúde e dos principais fatores de risco a mesma, que serão discutidos a seguir. 
Tabela 2

Estudos nacionais sobre a prevalência e severidade da fluorose dentária entre 1970 e 2000, segundo autor, ano de publicação, local, índice utilizado e concentração de flúor na água.

\begin{tabular}{|c|c|c|c|c|c|c|c|}
\hline Local & Autor, ano de publicação & Índice & $\mathrm{n}$ & Idade (anos) & $\begin{array}{c}\text { Prevalência } \\
\text { (\%) }\end{array}$ & $\begin{array}{l}\text { Moderado- } \\
\text { severos (\%) }\end{array}$ & $\begin{array}{l}\text { ppmF } \\
\text { na água }\end{array}$ \\
\hline Pereira Barreto (SP) & Uchôa \& Saliba (1970) & Dean & 442 & $6-15$ & 76,0 & 48,0 & $2,5-17,5$ \\
\hline Cosmópolis (SP) & Ando et al. (1973) & Dean & 175 & $6-14$ & 88,6 & 38,9 & $9,5-11,0$ \\
\hline Icém (SP) & Alcaide \& Veronezi (1979) & Dean & 449 & $7-14$ & 88,2 & 19,4 & $2,6-4,0$ \\
\hline Urussanga (SC) & Capella et al. (1989) & Dean & 338 & $3-10$ & 97,6 & 87,0 & $1,2-5,6$ \\
\hline Belo Horizonte (MG) & Silva \& Paiva (1995) & Dean & 518 & $7-14$ & 25,5 & 2,9 & $0,6-0,8$ \\
\hline Piracicaba (SP) & Gaspar et al. (1995) & Dean & 211 & $10-14$ & 20,9 & - & 0,7 \\
\hline Iracemápolis (SP) & Gaspar et al. (1995) & Dean & 200 & $10-14$ & 4,5 & - & 0,2 \\
\hline São Paulo (SP) & Narvai et al. (1997) & Dean & 2.491 & 12 & 21,8 & 3,7 & 0,8 \\
\hline Presidente Prudente (SP) & Buendia \& Zaina (1997) & Dean & 1.053 & $8-13$ & 17,5 & - & $0,8-1,0$ \\
\hline Curitiba (PR) & Alcântara (1998) & Dean & 360 & $7-14$ & 25,6 & 0,27 & 0,8 \\
\hline Araçatuba (SP) & Marcelino et al. (1999) & Dean & 317 & $12-14$ & 24,0 & 0,4 & 0,7 \\
\hline Osasco (SP) & Massaro $(2000)$ & Dean & 776 & $6-12$ & 49,4 & 3,6 & 0,8 \\
\hline Santos (SP) & Forni (2000) & Dean & 2.034 & $6-12$ & 49,2 & 2,5 & 0,7 \\
\hline São Vicente (SP) & Forni (2000) & Dean & 2.051 & $6-12$ & 54,8 & 0,4 & 0,7 \\
\hline Santo André (SP) & Forni (2000) & Dean & 922 & $6-12$ & 21,5 & - & 0,8 \\
\hline São Bernardo do Campo (SP) & Forni (2000) & Dean & 1.226 & $6-12$ & 19,7 & 1,5 & 0,8 \\
\hline São Caetano (SP) & Forni (2000) & Dean & 890 & $6-12$ & 59,4 & - & 0,8 \\
\hline Diadema (SP) & Forni (2000) & Dean & 449 & $6-12$ & 18,5 & 1,8 & 0,8 \\
\hline Mauá (SP) & Forni (2000) & Dean & 491 & $6-12$ & 42,6 & 0,8 & 0,8 \\
\hline Ribeirão Pires (SP) & Forni (2000) & Dean & 469 & $6-12$ & 84,0 & 0,4 & 0,8 \\
\hline Rio Grande da Serra (SP) & Forni (2000) & Dean & 370 & $6-12$ & 95,4 & 0,8 & 0,8 \\
\hline São Paulo (SP) & Forni (2000) & Dean & 776 & $6-12$ & 49,4 & 3,6 & 0,8 \\
\hline Itabaiana (PB) & Sampaio (1993) & TF & 437 & $6-14$ & 15,6 & - & $0,6-0,9$ \\
\hline São Félix (PB) & Sampaio (1993) & TF & 74 & $6-14$ & 10,8 & - & $0,6-0,7$ \\
\hline Mogeiro (PB) & Sampaio (1993) & TF & 98 & $6-14$ & 1,0 & - & 0,5 \\
\hline Piratininga (SP) & Tomita et al. (1995) & TF & 270 & $6-14$ & 34,4 & 2,2 & $0,5-1,3$ \\
\hline Olho D'Água (CE) & Cortes et al. (1996) & TF & 96 & $6-12$ & 91,7 & 18,8 & $2-3$ \\
\hline Vitória (ES) & Cortes et al. (1996) & TF & 201 & $6-12$ & 52,2 & 0,4 & 0,7 \\
\hline Maceió (AL) & Cortes et al. (1996) & TF & 160 & $6-12$ & 7,5 & - & 0,0 \\
\hline São Paulo (SP) & Adde (1997) & TF & 416 & $8-13$ & 15,1 & 3,2 & 0,8 \\
\hline Curitiba (PR) & SMS (1997) & $\mathrm{TF}$ & 380 & 12 & 55,3 & 0,3 & 0,9 \\
\hline Garça (SP) & Heintze et al. (1998) & TF & 206 & $5-24$ & 13,3 & - & 0,9 \\
\hline Bauru (SP) & Heintze et al. (1998) & TF & 167 & $5-24$ & 6,8 & - & 0,6 \\
\hline Itápolis (SP) & Heintze et al. (1998) & TF & 172 & $5-24$ & 1,7 & - & 0,0 \\
\hline Porto Alegre (RS) & Maltz \& Farias (1998) & TF & 135 & $8-9$ & 7,7 & - & 0,9 \\
\hline Arroio do Tigre (RS) & Maltz \& Farias (1998) & TF & 100 & $8-9$ & 0,0 & - & 0,2 \\
\hline Brasília (DF) & Maltz \& Farias (1998) & TF & 97 & $8-9$ & 22,3 & - & 0,8 \\
\hline Brasília (DF) & Campos et al. (1998) & TSIF & 833 & $8-12$ & 14,6 & - & 0,8 \\
\hline Luziânia (GO) & Maltz \& Farias (1998) & TF & 103 & $8-9$ & 2,1 & - & 0,2 \\
\hline Londrina (PR) & Gonini (1999) & TF & 434 & $9-12$ & 91,0 & 0,4 & $0,9-1,0$ \\
\hline São Paulo (SP) & Ribas et al. (1999) & TF & 553 & $6-12$ & 29,5 & - & 0,8 \\
\hline Vitória (ES) & Ferreira et al. (1999) & TSIF & 321 & $12-14$ & 70,8 & 1,7 & - \\
\hline
\end{tabular}

Siglas dos Estados: $\mathrm{AL}=$ Alagoas $; \mathrm{CE}=$ Ceará; $\mathrm{DF}=$ Distrito Federal $\mathrm{ES}=$ Espírito Santo; $\mathrm{GO}=$ Goiás; MG = Minas Gerais; $\mathrm{PB}=$ Paraíba; $\mathrm{PR}=$ Paraná; $\mathrm{RS}=$ Rio Grande do Sul; $\mathrm{SC}=$ Santa Catarina; $\mathrm{SP}$ = São Paulo. ppmf $=$ parte por milhão de fluoreto. 


\section{Fluorose dentária: principais fatores de risco}

\section{Formas tópicas de flúor}

Existem, atualmente, inúmeras fontes de exposição ao uso tópico de fluoretos no ambiente doméstico e profissional: dentifrícios, soluções para bochecho, géis e vernizes. A estes métodos atribui-se um porcentual significativo de redução da cárie dental, mas comportam-se como fatores de risco à fluorose em casos de deglutição, quando se constituem fontes sistêmicas da substância de alta concentração.

Diversos estudos demonstram associação positiva entre ingestão de dentifrícios na primeira infância e alta prevalência de fluorose dental (Brothwell \& Limeback, 1999; Warren \& Levy, 1999). Stookey (1994) estudando o uso de formas tópicas - dentifrícios e bochechos através de um estudo retrospectivo, identificou um odds de 2,73 vezes maior no caso de ingestão de soluções fluoretadas para bochechos, reafirmando a necessidade de se indicar cuidadosamente este método. Em relação ao uso tópico profissional, não foram encontradas associações significativas, provavelmente devido aos cuidados técnicos (uso de sucção, pequena quantidade, estímulo ao cuspir) envolvidos no processo (Horowitz,1995).

Como principais medidas recomendadas para limitar a toxicidade crônica das formas tópicas do flúor tem-se: a redução da concentração de flúor em pastas infantis, além de acrescentar nas embalagens instruções e precauções sobre seu uso em crianças; a necessidade da supervisão dos pais no momento da escovação, de forma a controlar a quantidade utilizada (máximo um grão de ervilha) de pasta dental e evitar a deglutição; contra-indicar o uso de soluções para bochechos em crianças menores de sete anos, bem como desenvolver ações efetivas de vigilância sanitária na mensuração da dosagem de flúor, indicada no produto pelos fabricantes (Brothwell \& Limeback, 1999; Horowitz, 1995; Levy et al., 1995; Stookey, 1994; Warren \& Levy, 1999).

\section{Fluoretação das águas}

de abastecimento público

Inúmeros são os estudos que descrevem associação positiva entre locais servidos com água de abastecimento fluoretada e a presença de fluorose dental no Brasil (Tabela 2) e no mundo. A prevalência da doença é diretamente proporcional à concentração da substância na água, e o risco atribuível referente a este méto- do varia entre $13,2 \%$ e $39,6 \%$ (Clarck et al., 1994; Heller et al., 1997; Jackson et al., 1995; Kumar \& Swango, 1999; Lewis \& Banting, 1994; Selwitz et al., 1995).

Além disso, também em regiões onde não há fluoretação das águas de abastecimento público, encontra-se aumento na ocorrência da fluorose, provavelmente decorrente, em parte, do "efeito halo" - bebidas e alimentos processados industrialmente em regiões com água fluoretada são distribuídos e consumidos em outros locais, difundindo o efeito do flúor em distintas populações (Horowitz, 1996; Lewis \& Banting, 1994; Selwitz et al., 1995).

No Brasil, existe uma cobertura desigual entre regiões com fluoretação das águas de abastecimento público (Tabela 3), embora para todo o país, esta seja semelhante ao encontrado em países como Estados Unidos e Reino Unido (Ferreira, 1998). Entretanto, já podem ser observados em municípios com e sem este benefício, prevalências maiores do que as esperadas de fluorose dental (Gonini, 1999; Maltz \& Farias, 1998; Mendonça et al., 1998).

Dentre os principais problemas no país, tem-se a dificuldade em manter sistemas operacionais de monitoramento dos níveis ótimos de flúor na água através do heterocontrole (Narvai, 2000), processo discutido desde o início da década de 80 no Estado de São Paulo, seja por falta de recursos humanos, técnico-operacionais ou de relevância para a comunidade. A maior parte dos trabalhos que acompanham a dosagem do nível ótimo de flúor em municípios brasileiros, descreve níveis irregulares de fornecimento do flúor, o que dificulta a avaliação da magnitude do efeito protetor à cárie dental e o risco à fluorose dental (Heintze et al., 1998; Modesto et al., 1999).

É inegável que a fluoretação das águas, apesar de ter sua efetividade reduzida com a difu-

Tabela 3

Cobertura da fluoretação das águas de abastecimento público. Brasil, 1996

\begin{tabular}{lcc}
\hline Regiões & \% água tratada & \% água fluoretada \\
\hline Norte & 42,30 & 13,13 \\
Nordeste & 50,29 & 6,85 \\
Sudeste & 66,89 & 61,48 \\
Sul & 70,24 & 65,39 \\
Centro-Oeste & 73,94 & 50,20 \\
Brasil & 61,30 & 42,09 \\
\hline
\end{tabular}

Fonte: Brasil, Ministério da Saúde, Coordenação de Saúde Bucal, 1997 (Ferreira, 1998) 
são dos fluoretos tópicos, continua a ser o método preventivo à cárie dental de mais ampla distribuição, maior eqüidade, adesão, melhor custo-efetividade e segurança (Horowitz, 1996; Lewis \& Banting, 1994). Apesar disso, deve ser tratado com cautela, e para isso, são fundamentais as ações de controle e pesquisa continuada sobre esse método, de forma a prevenir aumento na prevalência da fluorose dental sem reduzir o efeito protetor à cárie dental.

Dentre as medidas, pode-se citar: heterocontrole (Narvai, 2000) da dosagem ótima; monitoramento da prevalência da fluorose através de um sistema de informação consistente; ações de educação em saúde para toda a população, e desenvolvimento de equipamentos mais precisos e de fácil operação para análise dos teores de flúor (Kumar \& Swango, 1999; Modesto et al., 1999).

Suplementação com flúor (pastilhas, tabletes ou medicamentos)

O uso da suplementação sistêmica com flúor foi um método desenvolvido para a prevenção da cárie dental, substitutivo à fluoretação das águas de abastecimento público (Pendrys, 1995). Entretanto, muitas vezes é utilizado inadequadamente, concomitante a outros métodos sistêmicos ou em excesso de dosagem, constituindo-se num potencial fator de risco à fluorose dental nos primeiros oito anos de vida (Nowjack-Raymer, 1995; Pendrys, 1995; Wang, 1997).

Ismail \& Bandekar (1999) identificaram, numa meta-análise, que a maior parte dos estudos transversais e de caso controle, demonstra risco de 1,3 a 10,7 vezes maior de desenvolver fluorose dental quando submetidos à suplementação, em áreas com água não fluoretada. Em estudos longitudinais esse risco foi de 5 a 15 vezes maior nessa mesma associação.

Coloca-se então, a necessidade de cuidado adicional na indicação desse método: somente em crianças de alto risco - baixo nível sócioeconômico e educacional que dificulte o acesso a outros métodos tópicos; alto nível de ingestão de açúcares e filhos de mães com alta atividade/risco à cárie, e a populações isoladas. Além disso, é preciso amplo esclarecimento a outros profissionais de saúde sobre a disponibilidade de outros métodos sistêmicos de flúor oferecidos à comunidade (Horowitz, 1996; Ismail \& Bandekar, 1999; Levy, 1995; Nowjack-Raymer, 1995; Wang, 1997).

\section{Flúor ingerido na dieta}

Vários são os alimentos e bebidas disponíveis na alimentação que contêm alto teor de flúor e estão associados à presença da fluorose dental - peixes, mariscos, frango (quando alimentados com farelos de ossos) chás, além de bebidas, fórmulas infantis e leite quando processados em regiões com água de abastecimento público fluoretada (Clarck et al., 1994; Gonini, 1999; Horowitz, 1996; Levy, 1995).

Observa-se também, que é crescente o consumo, entre crianças, de alimentos industrializados, refrigerantes e leite em pó, acompanhados da redução do consumo de água e leite de outras fontes, o que aumenta significativamente a ingestão sistêmica de flúor numa idade de maior risco à fluorose. Entretanto, ainda hoje, é difícil medir a quantidade de ingestão de fluoretos pela dieta, por dificuldades metodológicas em mensurar níveis individuais de flúor de cada alimento na forma ativa, quantidade ingerida e total absorvido pelos tecidos (Brothwell \& Limeback, 1999; Heller, 1999).

Villena et al., (1996), num estudo realizado com 104 marcas comerciais de água mineral industrializadas no Brasil, descrevem que 7,7\% das mesmas continham níveis acima de $1 \mathrm{ppm}$ de flúor. É necessário, nesse sentido, reforçar as ações de vigilância em saúde, como a redução do teor de flúor em alimentos infantis manufaturados; normatizar a obrigatoriedade de rótulos que apresentem as concentrações de flúor, e que essas sejam feitas de forma padronizada (Levy, 1995; Villena et al., 1996) e ações de educação em saúde que possibilitem à população assimilar e interpretar as informações disponíveis (Horowitz, 1995).

\section{Conclusões}

A partir deste estudo pode-se concluir:

- Que a fluorose dentária constitui-se um relevante problema para a saúde bucal coletiva;

- Que a fluorose nas suas formas brandas são comuns onde há água de abastecimento público fluoretada, contribuindo para isso outras formas sistêmicas e tópicas do uso dos fluoretos;

- A forma severa da doença é observada, geralmente, em locais onde o flúor está presente em altas concentrações nos mananciais de água; - É necessário o heterocontrole da concentração adequada de flúor nos sistemas públicos de fornecimento de água;

- Da mesma forma, águas minerais, bebidas enlatadas, refrigerantes, sucos e chás devem ter o seu teor de flúor máximo permitido, regu- 
lamentado e apresentado em rótulos e embalagens, e com análise sistemática da vigilância sanitária;

- Como em outros estudos, reafirma-se a necessidade de reforçar a contra-indicação do uso de suplementos de flúor, além de outros medicamentos contendo a substância, em locais onde exista água de abastecimento fluoretada, de modo a evitar o excesso de ingestão sistêmica;

- Os cremes dentais devem ter os seus teores de flúor verificados periodicamente. A reco- mendação de reduzir a concentração de flúor nas pastas infantis deve ser discutida, bem como difundir a necessidade do uso de pequena quantidade de creme dental no ato de escovar os dentes, e a importância da supervisão dos pais ou responsáveis no momento da escovação, em crianças menores de sete anos;

- A necessidade de estudos epidemiológicos longitudinais no Brasil, de forma a acompanhar a tendência da prevalência e severidade da doença.

\section{Referências}

ADDE, C. A., 1997. Avaliação Crítica da Fluorose Dentária Quando da Associação de Métodos Sistêmicos e Tópicos de Fluoração em Escolares da Cidade de São Paulo, Após Dez Anos de Fluoração das Águas de Abastecimento Público. Tese de Doutorado, São Paulo: Faculdade de Odontologia, Universidade de São Paulo.

AKPATA, E. S.; FAKIHA, Z. \& KHAN, N., 1997. Dental fluorosis in 12-15 year old rural children exposed to fluorides from well drinking water in the Hail region of Saudi Arabia. Community Dentistry and Oral Epidemiology, 25:324-327.

ALCAIDE, A. L. \& VERONEZI, O., 1979. Prevalência de fluorose dental na cidade de Icém. Revista da Associação Paulista de Cirurgiões Dentistas, 33:2325.

ALCÂNTARA, C. M., 1998. Prevalência de fluorose dental em escolares de Curitiba. Revista Associação Brasileira de Odontologia - Nacional, 6:304307.

ANDO, T.; COIMBRA, B. W. R.; SANTOS, N. P. \& SAMPAIO, L., 1973. Efeito anticariogênico do flúor. Revista do Centro Acadêmico XXV de Janeiro/FOUSP, 32:7-11.

AZCURRA, A. I.; BATTELINO, L. J.; CALAMARI, S. E. \& CATTONI, S. T. D., 1995. Estado de salud bucodental de escolares residentes en localidades abastecidas con agua de consumo humano de muy alto y muy bajo contenido de fluorosos. Revista de Saúde Pública, 29:364-375.

BROTHWELL, D. J. \& LIMEBACK, A., 1999. Fluorosis risk in grade 2 students resinding in a rural area with widely varying natural fluoride. Community Dentistry and Oral Epidemiology, 26:130-136.

BUENDÍA, O. C. \& ZAINA, J., 1997. Será o flúor vilão? Revista Paulista de Odontologia, 19:36-44.
BURT, B. A., 1995. Introduction to the Symposium. Journal of Public Health Dentistry, 55:37-38.

CAMPOS, C.; TOLEDO, A. O. \& BEZERRA, A. C., 1998. Prevalência de fluorose dentária em escolares de Brasília - Distrito Federal. Revista de Odontologia da Universidade de São Paulo, 12:225-230.

CAPELLA, L. F.; CARCERERI, D. L.; PAIVA, S. M.; ROSSO, R. A.; PAIXÃO, R. F. \& SALTORI, E. K., 1989. Ocorrência de fluorose dentária endêmica. Revista Gaúcha de Odontologia, 36:371-375.

CLARCK, D. C. \& BERKOWITZ, Z. J., 1997. The influence of various fluoride exposures on prevalence of esthetic problems resulting from dental fluorosis. Journal of Public Health Dentistry, 57:144-149.

CLARCK, D. C.; HANN, H. J.; WILLIANSON, M. F. \& BERKOWITZ, Z. J., 1994. Influence of exposure to various fluoride technologies on the prevalence of dental fluorosis. Community Dentistry and Oral Epidemiology, 22:461-464.

CORTES, D. F.; ELLWOOD, R. P.; O'MULLANE, D. M. \& BASTOS, J. R., 1996. Drinking water fluoride levels, dental fluorosis and caries experience in Brazil. Journal of Public Health Dentistry, 56:226-228.

DENBESTEN, P. K., 1999. Biological mechanisms of dental fluorosis relevant to the use of fluoride supplements. Community Dentistry and Oral Epidemiology, 27:41-47.

DOWNER, M. C., 1994. Dental caries experience and defects of dental enamel among 12-year-old children in north London, Edinburgh, Glasgow and Dublin. Community Dentistry and Oral Epidemiology, 22:283-285.

EL NADEF, M. A. I. \& HONKALA, E., 1998. Fluorosis en relation to fluoride levels in central Nigéria. Community Dentistry and Oral Epidemiology, 26:2630. 
FEJERSKOV, O., 1994. Fluorose Dentária - Um Manual para Profissionais da Saúde. São Paulo: Editora Santos.

FEJERSKOV, O.; BAELUM, V.; MANJI, F. \& MOLLER, I. J., 1994. Fluorose Dentária. São Paulo: Editora Santos.

FERREIRA, H. C. G.; GOMES, A. M. M.; SILVA, K. R. C. S.; RODRIGUES, C. R. M. D. \& GOMES, A. A., 1999. Avaliação do teor de flúor na água de abastecimento público do município de Vitória - ES. Revista da Associação Paulista de Cirurgiões Dentistas, 53:455-459

FERREIRA, R. A., 1998. Em queda livre? Revista da Associação Paulista de Cirurgiões Dentistas, 52:52-55.

FORNI, T. I. B., 2000. Caracterização de Levantamentos Epidemiológicos de Fluorose Dentária no Estado de São Paulo, 2000. Dissertação de Mestrado, São Paulo: Faculdade de Saúde Pública, Universidade de São Paulo.

GASPAR, M. R.; ARMBRUSTER, L. M.; PEREIRA, A. C. \& MOREIRA, B. W., 1995. Opacidades de origem não fluorótica e fluorose dentária em áreas com baixa $(0,2 \mathrm{ppmF})$ e ótima $(0,7 \mathrm{ppmF})$. Revista Brasileira de Odontologia, 52:13-18.

GONINI, C. A. J., 1999. Fluorose Dentária em Crianças Nascidas entre 1986-1989, Usuárias da Rede de Unidades Básicas de Saúde de Londrina: Freqüência, Severidade e Fatores Associados. Dissertação de Mestrado, Londrina: Departamento de Saúde Coletiva, Universidade Estadual de Londrina.

HEINTEZ, S. D.; BASTOS, J. R. \& BASTOS, R., 1998. Urinary fluoride levels and prevalence of dental fluorosis in three Brazilian cities with different fluoride concentrations in the drinking water. Community Dentistry and Oral Epidemiology, 26:316-323.

HELLER, K. E., 1999. Water consumption in the United States in 1994-1996 and implications for water fluoritadion policy. Journal of Public Health Dentistry, 59:11-13.

HOROWITZ, H. S., 1995. Commentary on and recommendations for the proper use of fluoride. Journal of Public Health Dentistry, 55:57-62.

HOROWITZ, H. S., 1996. The effectiveness of community water fluoritadion in the United States. Journal of Public Health Dentistry, 56:253-258.

ISMAIL, A. I. \& BANDEKAR, R. R., 1999. Fluoride supplements and fluorosis: A meta-analysis. Community Dentistry and Oral Epidemiology, 27:48-56.

JACKSON, R. D., 1995. Dental fluorosis and caries prevalence in children residing in communities with different levels of fluoride in the water. Journal of Public Health Dentistry, 55:79-84.

KLOOS, H. \& HAIMANOT, R. T., 1999. Distribution of fluorosis in Ethiopia and prospects for control. Tropical Medicine \& International Health, 4:355364.

KLOOS, H.; HAIMANOT, R. T. \& JAHN, S. A. A., 1985. Water resources development and ecology in the Awash Valley, Ethiopia. Social Science and Medicine, 20:609-625.

KUMAR, J. V. \& SWANGO, P. A., 1999. Fluoride exposure and dental fluorosis in Newburgh and Kingston, New York: Policy implications. Community Dentistry and Oral Epidemiology, 27:171-180.
LEVY, S. M., 1995. Sources of fluoride intake in children. Journal of Public Health Dentistry, 55:39-52.

LEWIS, D. W. \& BANTING, D. W., 1994. Water fluoritadion: Current effectiveness and dental fluoroosis. Community Dentistry and Oral Epidemiology, 22:153-158.

LO, G. L. \& BAGRAMIAN, R. A., 1996. Prevalence of dental fluorosis in children in Singapore. Community Dentistry and Oral Epidemiology, 24:25-27.

MALTZ, M. E. \& FARIAS, C., 1998. Fluorose dentária em escolares de quatro cidades brasileiras com e sem água artificialmente fluoretada. Revista da Faculdade de Odontonlogia de Porto Alegre, 39:18-21.

MANDEL, I., 1992. Research in prevention of oral disease and conditions. Journal of Public Health Dentistry, 52:15-17.

MARCELINO, G.; GUIMARÃES, M. B.; SILVA, P. R.; TERRERI, A. L. M.; GUIMARÃES, L. O. C. \& SALIBA, N. A., 1999. Fluorose dentária em escolares da rede pública na cidade de Araçatuba. Revista do Instituto de Ciências da Saúde, 17:89-92.

MARTHALER, T. M., 1996, Water fluoritadion results in Basel since 1962: Health and political implications. Journal of Public Health Dentistry, 56:265270.

MASSARO, R. B. A., 2000. Prevalência da Fluorose Dentária em Pré-Escolares e Escolares Participantes do Programa de Promoção de Saúde Bucal da Prefeitura do Município de Osasco. Dissertação de Mestrado, São Paulo: Faculdade de Saúde Pública, Universidade de São Paulo.

MENDONÇA, L. L.; KISCHNER, U. L.; COSTA, R. N.; GIOVANNINI, J. F. G. B.; PINTO M. R. \& LUZ, M A. R., 1998. Estudo multicêntrico da fluorose dental e da cárie dental em escolares de 7 a 10 anos de Belo Horizonte-Parte I. Revista Pós Graduação-UFMG, 5:101-109.

MODESTO, A.; TANAKA, F. H. R.; FREITAS, A. D. \& CURY, J. A., 1999. Avaliação da concentração de fluoreto na água de abastecimento público do município de Rio de Janeiro. Revista Brasileira de Odontologia-ABO/RJ, 56:217-221.

NARVAI, P. C., 2000. Cárie dental e flúor: Uma relação do séc. XX. Ciência e Saúde Coletiva, 5:183189

NARVAI, P. C.; CASTELLANOS, R. A. \& FRAZÃO, P., 1997. Levantamento epidemiológico de oclusopatias e fluorose dentária em crianças de 5 a 12 anos de idade, no município de São Paulo, em 1996. In: II Congresso Brasileiro de Saúde Coletiva, Anais, p. 316. Águas de Lindóia: ABRASCO.

NEWBRUN, E., 1996. The fluoritadion war: A scientific dispute or a religious argument? Journal of Public Health Dentistry, 56:246-252.

N'ANG'A, P. M. \& VALDERHAUG, J., 1993. Prevalence and severity of dental fluorosis in primary schoolchildren in Nairobi, Kenya. Community Dentistry and Oral Epidemiology, 21:15-18.

NOWJACK-RAYMER, R. E., 1995. The prevalence of dental fluorosis in a school-based program of fluoride mouthrinsing, fluoride tablets and both procedures combined. Journal of Public Health Dentistry, 55:165-170.

PAIVA, S. M.; PAIXÃO, R. F.; CAPELLA, L. F.; CARCERERI, D. L.; ROSSO, D. A. \& FREITAS, A. R. R. 
1993. A fluorose dentária. Revista Gaúcha de Odontologia, 41:57-58.

PENDRYS, D. G., 1995. Fluoride supplements use by children in fluoridated communities. Journal of Public Health Dentistry, 55:160-164.

PINTO, V. G., 1994. Saúde Bucal, Odontologia Preventiva e Social. 4a Ed. São Paulo: Editora Santos.

RIBAS, T. R. C.; ARMONIA, P. L. \& SARACENI Jr., G., 1999. Avaliação da fluorose dentária em escolares do Colégio São Vicente de Paulo, situado na Zona Leste do município de São Paulo. Revista de Odontologia da Universidade de Santo Amaro, 4:62-65.

SAMPAIO, F. C., 1993. Prevalência de cárie e fluorose dentária em cidades da Paraíba com teores residuais de fluoretos na água de abastecimento. Centro de Ciências da Saúde, 12:9-11.

SELWITZ, R. H.; NOWJACK-RAYMER, R. E.; KINGMAN, A. \& DRISCOLL, W. S., 1995. Prevalence of dental caries and dental fluorosis in areas with optimal and above-optimal water fluoride concentrations:10 year follow up survey. Journal of Public Health Dentistry, 55:85-93.

SILVA, A. L. C. C. \& PAIVA, S. M., 1995. Ocorrência de fluorose dentária em escolares de Belo Horizonte. Revista do CROMG, 1:49-53.

SKOTOWSKI, M. C.; HUNT, R. J. \& STEVEN, M. L. 1996. Risk factors for dental fluorosis in pediatric dental patients. Journal of Public Health Dentistry, 55:154-159.
SMS-CURITIBA (Secretaria Municipal de Saúde), 1997. Fluorose - um estudo em crianças de 12 anos de Curitiba. Boletim Epidemiológico e Saúde Oral de Curitiba, 1:3-4.

STOOKEY, G. K., 1994. Review of fluorosis risk of selfapplied topical fluorides: Dentifricies, mouthrinses and gels. Community Dental and Oral Epidemiology, 22:181-186.

TOMITA, N. E.; PANIGHEL C. P. M. A; NARVAI, P. C. \& LOPES, E. C., 1995. Implicações da vigilância à saúde sobre ocorrência de fluorose dental. $R e$ vista da Associação Brasileira de Odontologia Nacional, 3:318-323.

UCHÔA, H. W. \& SALIBA, N. A., 1970. Prevalência de fluorose dental na cidade de Pereira Barretos. Boletim do Serviço de Odontologia Sanitária da Secretaria de Saúde, 6:11-16.

VILLENA, R. S.; CURY, J. A. \& BASTOS, R., 1996. Avaliação da concentração de flúor de águas minerais comercializadas no Brasil. Revista de Saúde Pública, 30:512-518.

WANG, N. J., 1997. Risk factors associated with fluorosis in a non-fluoridated population in Norway. Community Dental and Oral Epidemiology, 25: 396-402.

WARREN, J. J. \& LEVY, S. M., 1999. A review of fluoride dentifrice related to dental fluorosis. Pediatric Denistry, 21:265-271.

Recebido em 29 de janeiro de 2001

Versão final reapresentada em 7 de maio de 2001

Aprovado em 25 de julho de 2001 